activation, management of non-severe impairment, and when to begin work activation were locations of developing and disparate policies.

Conclusion A relationship between reduced number of people on disability benefits and increased employment is not always clear. More tracking is needed of employment outcomes for people who receive or are declined support. Strategies for successfully engaging employers and health care providers in work integration are still developing. Areas of emergent concern are effects of quality of the claimant encounter with providers and approaches to support the growing self-employment economy.

\section{DEVELOPMENT OF AN INTERVENTION TO ENHANCE SELF-MANAGEMENT AT WORK FOR WORKERS WITH A CHRONIC DISEASE USING INTERVENTION MAPPING}

AR Bosma*, CRL Boot, FG Schaafsma, JR Anema. VU University Medical Centre, Amsterdam Public Health research institute, department of Public and Occupational Health, Amsterdam, The Netherlands

\subsection{6/oemed-2018-ICOHabstracts. 1541}

Introduction The number of people in the working population diagnosed with one or more chronic diseases will increase. Self-management at work may facilitate workers with a chronic disease to continue working. Occupational and insurance physicians can play a key role in supporting this selfmanagement at work, as this fits within their preventive task. The aim of this project is to explore how occupational and insurance physicians can optimally support chronically ill workers in improving their self-management at work. We will develop an intervention that will fit within the needs of all stakeholders involved, using Intervention Mapping (IM) as a tool.

Methods The intervention will be developed with the use of the 6 step IM protocol:

- Needs assessment;

- Definition of change objectives;

- Selection of theory based methods and practical strategies;

- Program development;

- Adoption and implementation plan;

- Evaluation plan.

Step 1, the needs assessment will consist of a systematic review of the literature and a qualitative explorative study using focus groups. In the focus groups with chronically ill workers, occupational and insurance physicians we will explore their perceptions on self-management at work, their needs for support in improving self-management at work and the views of occupational and insurance physicians on providing this support.

Result The results of the needs assessment are expected early 2018 and will be available for presentation at the conference. Discussion The iterative steps of the IM protocol help to develop an intervention for occupational and insurance physicians that enables them to support workers with a chronic disease in improving self-management at work, to maintain productivity and to prevent sick leave and job loss.

\section{4 PERSON-RELATED FACTORS ASSOCIATED WITH WORK PARTICIPATION IN EMPLOYEES WITH HEALTH PROBLEMS: A SYSTEMATIC REVIEW}

M de Wit*, H Wind, CTJ Hulshof, MHW Frings-Dresen. Academic Medical Centre, department Coronel Institute of Occupational Health, Amsterdam Public Health research institute, Amsterdam, The Netherlands

\subsection{6/oemed-2018-ICOHabstracts. 1542}

Introduction Sick leave or work disability, is a major health problem, with negative consequences for the employee, employer and society. In order to prevent or decrease the duration of sick leave, it is important to know which factors influence work participation. Research revealed that besides disease-related factors, person-related factors like cognitions and perceptions of employees play a role in work retention and return to work after sick leave. Structured information about these factors can help occupational- and insurance physicians in their activities to decrease the duration of sick leave. However, structured information about the influence of person-related factors on work participation of workers with health problems is not available yet. This study aims to provide systematically assessed information about the association between person-related factors and work participation.

Methods A systematic review was conducted to explore the association between person-related factors and work participation. PubMed and PsycINFO were used to search for papers published between January 2007 and February 2017. There were no restrictions in study design. The methodological quality of included studies was assessed using quality assessment tools of the Joanna Briggs Institute.

Result 116 of 3465 studies were included which addressed the association between person-related factors and work participation. Evidence was found for a positive association between motivation, positive recovery expectations, self-efficacy, intern locus of control, perceived health, and optimism and work retention or return to work after sick leave. The personrelated factors catastrophizing, fear-avoidance coping, and perceiving work as a cause of the disability were negatively associated with work retention or return to work after sick leave. Discussion The results provide input for developing tailored interventions and provide guidance for occupational- and insurance physicians to facilitate returning to work and work retention. Further research is required to determine how these physicians could acquire and apply information about person-related factors.

\section{CHARACTERISTICS OF INDIVIDUALS RECEIVING DISABILITY BENEFITS IN THE NETHERLANDS AND PREDICTORS OF LEAVING THE BENEFIT SCHEME. A FIVE-YEAR FOLLOW-UP STUDY} ${ }^{1,2,3}$ I Louwerse*, ${ }^{1,3} \mathrm{MA}$ Huysmans, ${ }^{1,2} \mathrm{HJ}$ van Rijssen, ${ }^{1,3} \mathrm{AJ}$ van der Beek, ${ }^{1,3} \mathrm{JR}$ Anema.
${ }^{1}$ Department of Public and Occupational health, VU University Medical Centre, Amsterdam,
The Netherlands; ${ }^{2}$ Dutch Institute of Employee Benefit Schemes (UWV), Amsterdam, The
Netherlands; ${ }^{3}$ Research Centre for Insurance Medicine, AMC-UMCG-VUmC, Amsterdam,
The Netherlands

\subsection{6/oemed-2018-ICOHabstracts. 1543}

Introduction Nowadays, work disability is one of the biggest social and labour market challenges for policy makers in 
almost all OECD countries. Understanding of factors associated with long-term work disability may be helpful to identify groups of individuals at risk for disability benefit entitlement or continuing eligibility, and to develop effective interventions for these groups. The purpose of this study is to give insight into the main diagnoses of workers who qualify for disability benefit and how these diagnoses differ between age groups, gender and educational level. Moreover, using a 5 year follow-up period, we study the duration of the disability benefit and examine how durations differ between individuals with different characteristics.

Methods Our study population consisted of 31733 individuals receiving a disability benefit from the Dutch Social Security Agency (SSA). Data were collected from the databases of the SSA. Disorders were assessed by an insurance physician at application. We tested for differences in socio-demographics, main diagnoses and comorbidity for those entering and leaving disability benefits. Result Mental disorders were most often registered as the main diagnosis for work disability. Diagnoses differed between age groups and educational level categories. For younger and higher educated individuals mental disorders was the main diagnosis for work disability, and for older and lower educated individuals physical disorders (mainly musculoskeletal, cardiovascular and cancer). Five years after approval, 82\% still received disability benefits. Outflow was lowest for individuals with (multiple) mental disorders and individuals with comorbidity of mental and physical disorders, and highest for individuals with (multiple) physical disorders.

Conclusion The main diagnosis for persons entitled to disability benefits was mental health problems, especially for young women. In a five-year follow-up, claim duration for disability benefits was long lasting for most claimants.

\section{ADHERENCE TO VOICE THERAPY RECOMMENDATIONS IS ASSOCIATED WITH PRESERVED FITNESS-FOR-WORK AMONG TEACHERS WITH OCCUPATIONAL VOICE DISORDERS}

1,2 Lilah Rinsky-Halivni* ${ }^{1}$ Miriam Klebanov ${ }^{3}$ Yehuda Lerman, ${ }^{2}$ Ora Paltiel. ${ }^{1}$ Department of Occupational Medicine, Jerusalem District, Clalit Health Services, Jerusalem; ${ }^{2}$ Braun School of Public Health, Hadassah-Hebrew University Medical Centre, Jerusalem, Israel; ${ }^{3}$ School of Public Health, Sackler Faculty of Medicine, Tel Aviv University, Tel Aviv, Israel

\subsection{6/oemed-2018-ICOHabstracts.1544}

Introduction Vocal problems among teachers are considered a common professional hazard and a significant source of disability, leading to reductions in quality-of-life and professional capacities. Factors related to fitness-for-work reductions were not evaluated so far. Recommendations for voice-therapy, rest and microphone use are common interventions in occupational medicine aimed at preserving the working capability of these teachers and reducing work disability. Research on the impact of such interventions on employment capacity is lacking. The aims of the study regarding dysphonic teachers referred to occupational medicine clinic: evaluate employment outcomes following voice-therapy, voice rest and microphone use, and identify other predictors to limitations in fitness-for-work.

Methods Teachers who were first referred to occupational medicine clinic due to voice disorders between 1/2007 and 12/2012 were followed-up. Data were collected from medical records and from interviews conducted in 2014. Logistic regression models were utilised to assess associations between interventions and other covariates, and employment outcomes. Survival analysis was performed to evaluate association between undergoing voice-therapy and length of retained fitness-for-work.

Results A sample of 153 dysphonic teachers comprised the prospective study cohort, which was followed for 2-8 years. Thirty-four $(22.2 \%)$ suffered declines in working capabilities. Voice-therapy was protective against such declines $[\mathrm{OR}=0.05$ (0.01-0.27)], in contrast to voice rest and microphone use. Compliance with voice-therapy recommendation was less than $50 \%$. Most of declines in fitness-for-work among non-compliant teachers occurred within 20 months after referral. Other predictors were associated with work fitness reduction.

Discussion Voice-therapy, especially when instituted early, is the strongest predictor for retaining fitness-for-work among dysphonic teachers. Pilot study with employers is warranted to estimate compliance and economic feasibility of preventive measures including voice-therapy aimed at reducing disability and preserving work capacities.

\section{RETURN TO WORK OF CANCER PATIENTS AFTER A MULTIDISCIPLINARY INTERVENTION INCLUDING OCCUPATIONAL COUNSELLING AND PHYSICAL EXERCISE: A PROSPECTIVE STUDY}

${ }^{1}$ Angela GEM de Boer* ${ }^{1}$ Monique CJ Leensen, ${ }^{2}$ Iris F Groeneveld, ${ }^{1}$ Iris van der Heide, ${ }^{3}$ Tomas Rejda, ${ }^{4}$ Peter $\amalg$ van Veldhoven, ${ }^{5}$ Sietske van Berkel, ${ }^{5}$ Aernout Snoek, ${ }^{6,7,8}$ Wim van Harten, ${ }^{1}$ Monique HW Frings-Dresen. ${ }^{1}$ Coronel Institute of Occupational Health, Academic Medical Centre, Amsterdam Public Health research institute, Amsterdam, The Netherlands; ${ }^{2}$ Rijnlands Rehabilitation Centre, Leiden, The Netherlands; ${ }^{3}$ Leiden University Medical Centre, Leiden, The Netherlands; ${ }^{4}$ Department of Sports Medicine, Haaglanden Medical Centre, The Hague, The Netherlands; ${ }^{5}$ Department of Sports Medicine, Isala, Zwolle, The Netherlands; ${ }^{6}$ University Twente, Enschede, The Netherlands; ${ }^{7}$ Rijnstate Hospital, Arnhem, The Netherlands; ${ }^{8}$ The Netherlands Cancer Institute, Amsterdam, The Netherlands

\subsection{6/oemed-2018-ICOHabstracts. 1545}

Introduction To support return to work among cancer patients, a multidisciplinary rehabilitation program was developed which combined occupational counselling with a supervised physical exercise program during chemotherapy. Aim was to investigate return to work (RTW) rates of cancer patients and to evaluate changes in work-related, quality of life and physical outcomes.

Methods This was a longitudinal prospective intervention study using a one-group design. Patients with primary diagnosis of cancer from two medical centres were included if they were treated curatively with chemotherapy and on sick leave from paid work. The 12 week multidisciplinary rehabilitation program combined occupational counselling with a supervised physical exercise program. Patients completed questionnaires on RTW, importance of work, work ability (WAI), RTW selfefficacy, fatigue (MFI), and quality of life (EORTC QLQ C30) at baseline and 6, 12 and 18 months follow-up. Before and after the exercise program 1-Repetition Maximum (RM) muscle strength and cardiorespiratory fitness $\left(\mathrm{VO}_{2}\right.$ peak) were assessed.

Result 93 Patients were included. Six months after the start, $59 \%$ of the cancer patients returned to work, $86 \%$ at 12 months and $83 \%$ at 18 months. In addition, significant improvements $(\mathrm{p}<0.05)$ in importance of work, work ability, RTW self-efficacy, and quality of life were observed, whereas fatigue levels were significantly reduced. After completing the exercise program, 1-RM muscle strength was significantly increased but there was no improvement in $\mathrm{VO}_{2}$ peak. 